JGG 2020;68:216-223

doi: 10.36150/2499-6564-265

\title{
What is known in male gender differences, comorbidity and age for COVID-19 pandemia? A narrative minireview
}

\author{
Tiziana Ciarambino ${ }^{1}$, Gennaro Sansone ${ }^{2}$, Giovanni Menna², \\ Ombretta Para ${ }^{3}$, Mauro Giordano² \\ ${ }^{1}$ Internal Emergency Department, Hospital of Marcianise, ASL Caserta, Italy; ${ }^{2}$ Department of Medical \\ science, University of Campania, L. Vanvitelli, Naples, Italy; ${ }^{3}$ Internal Emergency Department, Careggi \\ Hospital University of Florence, Italy
}

Background. On March 2020, WHO declares the world pandemic by COVID-19. In this report we report the COVID-19 infection, related to male gender, comorbidity and special population.

Data resources. We describe the published studies by PubMed, Medscape and Scopus between December 2019 to May 2020. Keywords used: male/man gender, sex differences, COVID-19, comorbidity, diabetes, hypertension, elderly, pregnancy, children.

Results. The elderly population and infants are a population at higher risk. The comorbidities are risk factors for the development of a more severe form of disease. There may be a sex predisposition to COVID-19 infection, with men more prone to be affected. $83.9 \%$ of COVID-19 patients with chronic kidney disease (CKD) and 57.3\% of COVID-19 patients with liver diseases, have a severe disease.

Received: September 14, 2020

Accepted: November 16, 2020

\section{Correspondence}

Tiziana Ciarambino

PO Marcianise, ASL Caserta, via Orto dell'Abate, 81025 Marcianise (CE), Italy

E-mail: tiziana.ciarambino@gmail.com

\section{Conflict of interest}

The Authors declare no conflict of interest

How to cite this article: Ciarambino T, Sansone G, Menna G, et al. What is known in male gender differences, comorbidity and age for COVID-19 pandemia? A narrative minireview. Journal of Gerontology and Geriatrics 2020;68:216-23. https://doi.org/10.36150/2499-6564-265

(C) Copyright by Società Italiana di Gerontologia e Geriatria (SIGG)

\section{(c) (1) () $\odot$}

\section{OPEN ACCESS}

This is an open access article distributed in accordance with the CC-BY-NC-ND (Creative Commons Attribution-NonCommercial-NoDerivatives 4.0 International) license. The article can be used by giving appropriate credit and mentioning the license, but only for non-commercial purposes and only in the original version. For further information: https://creativecommons.org/licenses/by-nc-nd/4.0/deed.en
Conclusions. Older age, infants, male gender and comorbidity describe a crucial role for severity of COVID-19 disease. Future studies are need for the management of these patients.

Key words: male gender, man differences, COVID-19, special population, comorbidity, diabetes, hypertension, elderly, pregnancy, children

\section{INTRODUCTION}

A new coronavirus in late 2019 resulted in a number of pneumonia cases in China in people who attended the Wuhan fish market in the Hubei region ${ }^{1}$. This virus quickly spreads to numerous other countries, in February 2020 $\mathrm{WHO}$ defines the disease triggered by this coronavirus: COVID-19 ${ }^{2,3}$. On 11 March 2020, WHO declares the world pandemic, with 118,000 cases in 114 countries and 4.291 deaths from COVID-19 ${ }^{4}$. The virus that causes COVID-19 is a virus of the genus Beta-coronavirus and of the species SARS-CoV (severe acute respiratory syndrome-related coronavirus), and is called SARS-CoV-2 ${ }^{5}$. The SARS-CoV-2 virus uses the ACE2 receptor (angiotensin-converting enzyme-2) to enter into the cells of the upper respiratory tract ${ }^{1}$. The aim of this review is to analyze the gender differences, comorbidities and the special population related to COVID-19.

\section{METHODS}

The included studies were identified by using PubMed, Medscape and 
Scopus on the special population, male gender and COVID-19 until $30^{\text {th }}$ June 2020. Keywords used: male gender, man differences, COVID-19, special population, comorbidity, diabetes, hypertension, elderly, pregnancy, children. Clinical trials, retrospective and prospective studies were included. Studies written in languages other than English were excluded. Three authors (T.C., G.S. and G.M.) reviewed all study abstracts. Studies were included if gender differences in comorbidity and COVID-19 were reported. All selected studies were qualitatively analyzed.

\section{SPECIAL POPULATION}

\section{Elderly population}

The elderly is certainly the most fragile population, which has been most exposed to COVID-19. In fact, in Italy, $39.1 \%$ of infected patients were over 70 years old and $72.2 \%$ were over 50 years old. In addition, elderly have a markedly higher mortality than the young ${ }^{6,7}$. A study of 1099 patients with SARS-CoV-2 showed that $15.1 \%$ of the population is 60 years old and above, and $27 \%$ of those who are critically is 60 years old and above. Another large study analyzed 4021 confirmed cases, and 1052 (26.2\%) were 60 years old or older. The mortality rate of patients aged 60 years old and over $(5.3 \%)$ is significantly higher than that of patients under 60 years old $(1.4 \%)^{8}$. There are important correlations between patient's age and inflammatory indicators, with lower levels of lymphocyte counts and albumin in patients over 60 years old. On other hand, levels of lactate dehydrogenase (LDH), blood urea nitrogen and C-reactive protein (CRP) are higher in patients over 60 years old and there are even more pulmonary lobes involved. However, it has been reported a higher proportion of bacteria co-infection in patient over 60 years old compared to those younger than $60{ }^{9}$. According to the report of 72.314 cases from the Chinese Centre for Disease Control and Prevention, the case fatality rate (CFR) was significantly higher in older and geriatric patients. In particular, it has been reported that the CFR was of $2.3 \%$ in all confirmed cases, $8.0 \%$ in patients aged $70-79$ years old and $14.8 \%$ in patients over 80 years old ${ }^{10}$. So, the elderly population is at higher risk, and these data are probably related to the greater presence of comorbidities such as hypertension, diabetes, COPD, heart disease and chronic kidney disease. In particular, in the Centre for Disease Control data, hypertension is present in $63.1 \%$ of adults older than 60 , chronic kidney disease (CKD) in 38\% of adults older than 60 , and diabetes in $26.8 \%$ of adults older than age $65^{11}$. These are risk factors for the development of a more severe form of disease, and therefore in this population it has been reported a higher rate of ARDS and complications related to COVID-19 ${ }^{12-14}$. To this regard, it has been reported that, in elderly or geriatric patients, the cardiac injury due to myocardial damage caused by SARS-CoV-2, induce more fatal outcomes and higher mortality than those without ${ }^{9}$. In addition, the CFR in patients without comorbidities was $1.4 \%$, whereas it was $13.2 \%$ for patients with cardiovascular disease, 9.2\% for patients with diabetes, $8.4 \%$ for patients with hypertension, $8 \%$ for patients with chronic respiratory disease, and $7.6 \%$ for patients with cancer ${ }^{11}$.

However, other two elements characterized the elderly during this pandemic: social isolation and malnutrition. Social isolation for older adults is a "serious public health concern" because of their risk of cardiovascular, autoimmune, neurocognitive, and mental health problems ${ }^{14}$. Some studies demonstrated that social isolation puts older adults at greater risk of depression and anxiety ${ }^{15}$. On one hand, it is important for elderly people to remain home, with food and medications delivered, and avoid social contact with family and friends, on other hand, it is even important to have actions to mitigate the mental and physical health consequences ${ }^{14}$. For what concern malnutrition, a study reported that incidence of malnutrition in the elderly was about $50.5 \%$ higher in rehabilitation institutions, and $38.7 \%$ in hospitalized patients. However, the incidence of malnutrition in elderly patients with COVID-19 seems to be higher, and this phenomenon certainly participates in the development of immune dysregulation, which could account for a greater susceptibility to Covid-19 for elderly people ${ }^{16,17}$.

\section{Children population}

Children of all ages can be infected, from newborn infants to young children. In the largest Chinese pediatric case, the median age at pediatric diagnosis was seven years. Instead, the median was 6.7 years in 171 patients from the Wuhan Children's Hospital ${ }^{18}$. The rate of incidence in children for COVID-19 is low, accounting for about $2 \%$ in China ${ }^{19}$. Similar findings were observed in Europe with $1 \%$ in children less than 10 years old and $4 \%$ in 10 to 19 years old. Fever and cough are the commonest presentation in symptomatic cases. Radiological imaging is abnormal in more than half of cases. All cases in children recovered don't reported fatality ${ }^{19}$. In fact, adult and elderly patients are more prone to end-organ damage, systemic failures and death following COVID-19 than children. The explanation could be related to the impaired immunity in the elderly patients, and the increased prevalence of co-morbidities such as cancer, diabetes and cardiovascular diseases in the adult and in the elderly ${ }^{19}$. There are other theories underlying this epidemiological difference between adults and children. Some studies show the possibility 
of the presence of more antibodies against viruses and of an immune system differently responsive for children than adults. Other studies propose the possibility of a reduced presence of the ACE2 receptor on the cells of the respiratory tract of children ${ }^{20}$. In addition, it should be highlighted that infants could have worse complications than children ${ }^{20}$. Most of the children patients manifested mild symptoms. There are several risk factors for severity in children who were hospitalized with COVID-19. For laboratory tests, the presence of elevated white blood cell, high CRP, and activated IL-6, IL-10, NK and the higher INR, D-dimer are risk factors for higher severity ${ }^{21}$. Moreover, regarding radiological findings, in this study ${ }^{21}$ it has been reported, that at the first day of admission, severe children patients manifested computerize tomography (CT) image positive associated with COVID-19 disease severity ${ }^{21}$. The most vulnerable fragments in these patients are left lower lung, right upper lung, right lower lung, rather than just lower lobe. In adult, conversely, is damaged often lower lobe ${ }^{21}$. A study suggest that more than three lung segments was the high-risk factor associated with severe COVID-19 in children ${ }^{21}$. In a Chinese study, laboratory and radiological characteristics, were analyzed in 20 children affected by COVID-19 and they found that, in 16 of 20 children, the Procalcitonin level was high; 12 of 20 children had ground glass opacity at the chest CT scan; and in 10 of 20 children, in chest CT scan there was an opacity surrounded by a halo sign, that could be a typical sign of COVID-19 in children. Also, in 8 of 20 children was found a bacterial co-infection, which is probably more common in children than in adult ${ }^{22}$. For the children who need admission to hospital, the management is based on supportive therapy, including adequate nutrition and calorie intake, fluid and electrolyte management and oxygen supplementation. Another important issue of management is the communication with parents with the aim of alleviating anxiety ${ }^{23}$. Finally, a multisystem inflammation syndrome has been found in children with COVID-19, which could clinically appear very similar to Kawasaki disease. Indeed, in Italy a cluster of ten children showed symptoms very similar to Kawasaki (i.e., non-purulent conjunctivitis, polymorphic rash, mucosal changes, and swollen extremities) ${ }^{24-26}$. About newborn, if the puerperant is positive for SARSCoV-2, the neonate must be isolated, then detected SARS-CoV-2, because an early identification and isolation are very important for COVID-19 control ${ }^{27}$. COVID-19 neonates should be placed in negative pressure rooms ${ }^{27}$. In conclusion, the management of COVID-19 newborns with severe acute respiratory distress syndrome (ARDS), is the same of all cause of ARDS and neonatologist could use high-dose pulmonary surfactant, inhaled nitric oxide, high-frequency oscillatory ventilation, and extracorporeal membrane lung may be useful ${ }^{27}$.

\section{Pregnant women}

The data related to SARS infection in pregnant women showed a high incidence of adverse maternal and neonatal complications, such as spontaneous miscarriage, preterm delivery, intrauterine growth restriction, endotracheal intubation, admission to the intensive care unit, renal failure, and disseminated intravascular coagulopathy. On the other hands, during the COVID-19 disease, the course of infection of pregnant patients tends to be similar to non-pregnant women ${ }^{28}$. Pregnancy is a very special time in a woman's life and the response to inflammation also changes from trimester to trimester. Indeed, the first trimester is pro-inflammatory, the second is anti-inflammatory, and the third trimester is a pro-inflammatory phase again. Coronavirus is a pro-inflammatory disease, therefore for the reasons mentioned above, pregnant women are at higher risk of contracting the disease in the first and third trimester ${ }^{29}$. Phoswa et al., suggest that pregnancy could be a risk factor for the development of COVID-19, because pregnant women showed decreased lymphocytes and Natural Killer cells, increased NKG2A inhibitory receptors, and increased ACE2, IL-8, IL-10 and IP-10 levels. However, these changes in immune response are similar to those that occur during COVID-19 infection, for this reason the authors describe that pregnancy could be a risk factor for COVID-19 ${ }^{29}$. For the radiological diagnosis of COVID-19, X-ray and CT scan, should be used even for pregnant women as clinically necessary, with informed consent obtained, abdominal shielding and limiting exposure times to the minimum necessary ${ }^{30}$. Chest CT features of COVID-19, in pregnant patients, are the same of non-pregnant patients. Indeed, it has been reported, ground glass opacity with consolidation or reticulation, and complete consolidation with predominantly peripheral distribution and bilateral lung involvement. However, the consolidation lesions seem to be more prevalent in the pregnant cases ${ }^{31}$. The placenta of pregnant women with COVID-19 infection, should be considered as biohazardous waste ${ }^{30}$. If the maternal COVID-19 infection, is assessed as severe or critical, early delivery should be considered to ensure maternal safety, regardless of gestational age ${ }^{32}$. Some studies have even analyzed the possibility of the transplacental passage of SARS-CoV-2, but it was considered unlikely ${ }^{33}$. Regarding breast-feeding, limited data showed that SARS-CoV-2 is not transmitted through breast milk, but further studies are needed ${ }^{34}$. Furthermore, the complications related to the mental health of pregnant women during the lockdown for the pandemic, must not be overlooked. In fact, in many cities in the United 
States, the pregnant women had to deliver without their partner or relatives, and women with COVID-19 were immediately separated from the newborn in order to reduce the infection. These elements and the need for social distance could make pregnant and post-partum women more vulnerable and isolated ${ }^{35}$.

\section{Male and female subjects}

In China and in Italy men are more infected by SARSCoV-2 than women ${ }^{36}$. Several studies point out that men are more predisposed to develop Covid-19, and, in a study realized in New York, $70.8 \%$ of patients in invasive mechanical ventilation were men ${ }^{37-39}$. Study about gender distribution, suggested that males account for $60 \%$ of COVID-19 patients. Reasons are not well explored yet. Some studies showed that even MERS-CoV and SARS-CoV infected more males than females. Many factors can explain this disparity. In particular, pre-existing diseases, such as cardiovascular or respiratory impairment, hypertension or diabetes may unfavorably impact the course of the COVID-19. However, higher risk behaviors, such as alcohol abuse and smoking, more common in male than female, may play a role in the pathophysiological process of COVID-19 ${ }^{40,41}$. However, it has been reported that hormones such as sex-specific steroids (estrogens, androgens and progesterone), and activity of X-linked genes, exert different effects on immune regulation ${ }^{42}$. In fact, these modulate the innate and adaptive immune response to virus infection and influence the immune response ${ }^{36,43}$.

It is well known that estrogen suppresses $T$ and $B$ cell lymphopoiesis, activates B cell function and influences $\mathrm{T}$ cell development. Moreover, estrogen regulates a number of cytokines (such as IL-1, IL 10, and IFN- $\gamma$ ) that modulate the immune response. While estrogen has immune-stimulatory roles, progesterone and androgens are immune-suppressive and counteract the pathways affected by estrogen ${ }^{44}$. In particular, progesterone increased IL-4, reduced IFN- $\gamma$ (Th17) responses and reduced $\mathrm{T}$ cell proliferation and $\mathrm{T}$ cell dependent antibody responses. However, in CD8 T cells, progesterone reduced IFN- $\gamma$ and cytotoxicity ${ }^{45}$. Other possible explanation about these differences between male and female subjects could be related to a higher number and activity of innate immune cells (including monocytes, macrophages, and dendritic cells, higher basal levels immunoglobulin and antibody responses) in women.

$X$ chromosome contains several immune-related genes. In particular, females are mosaics for X-linked genes, and this contribute to generate a stronger immune response (both innate and adaptive) and more frequent autoimmune and inflammatory diseases in female subjects ${ }^{46}$. However, ACE-2 represents the primary route of infection of COVID-19 and it is located on $\mathrm{X}$ chromosome. It is expressed in lungs, kidneys, myocardium, gastrointestinal system and reproductive organs. Actions exerted by this enzyme consist not only in the conversion of Angiotensin I, but also in immunomodulation and prevention of lung injury, with a protective effect in female subjects ${ }^{47}$.

However, there are even behaviors which could contribute to a higher incidence for men, such as lower rates of hand washing, which is a recognized preventative measure, and delayed healthcare seeking ${ }^{49}$. Gender differences are reported in symptoms by COVID-19. To this regard, females are more affected by hyposmia or anosmia and gustatory dysfunction compared to males ${ }^{36}$. As for hospitalization rates, men are more hospitalized than women, and even the number of intensive care unit (ICU) admissions are higher in men ${ }^{49}$. These data appear to indicate that there may be a sex predisposition to Covid-19, with men more prone to be affected ${ }^{50}$. In addition, Liu et al., analyzed post-traumatic stress symptoms (PTSS) prevalence in gender. This study demonstrated higher symptom prevalence in females, and women suffer more re-experiencing, negative alterations in cognition or mood and hyperarousal ${ }^{51}$.

\section{COMORBIDITY}

\section{Chronic kidney disease and liver dysfunction}

In a study on April 2020, the prognosis of Covid-19 patients with chronic kidney disease (CKD) and liver pathologies is analyzed. The authors indicate that the prevalence of CKD and liver pathologies is 1 and $3 \%$, respectively, in COVID-19 patients ${ }^{52}$. In particular, 83.9\% of COVID-19 patients with CKD, have a severe form of disease, while $57.3 \%$ of COVID-19 patients with liver diseases, have a severe form. The mortality in these two groups of patients is $53.3 \%$ in patients with CKD, and $17.6 \%$ in patients with liver pathologies ${ }^{52}$.

Despite this, it has not been unequivocally documented that pre-existing liver diseases can modify the course of the COVID-19 disease, but it is universally accepted that there is an increase in markers of liver damage in patients with COVID-19. However, the cause of this damage is not known ${ }^{53}$. Some authors report that the virus was not found in the liver in autopsy findings, probably because the ACE2 receptor is not present on the hepatocyte membrane, and therefore liver damage could be linked to drug toxicity, cytokine storm or hypoxia ${ }^{52}$. Other authors, conversely, report that in $48 \%$ of patients, the virus is in their stool, even after the negativity of nasopharyngeal sample, and this may be evidence of the presence of the virus in the portal 
circulation. Furthermore, they reported the presence of the ACE2 receptor mainly in cholangiocytes, but also to a lesser extent in hepatocytes. This, in their view, could support the hypothesis of direct cytotoxic damage of the virus. Furthermore, given the presence of numerous inflammatory cells, they do not exclude the possibility of immune-mediated liver damage ${ }^{54}$.

ACE2 receptors seem to be over-expressed on tubular cells and dysregulation of the immune system is classically reported in patients with CKD. In particular, they have an altered ratio of $\mathrm{CD} 4^{+} / \mathrm{CD} 8^{+} \mathrm{T}$ lymphocytes and a lower activity of natural killer cells. It should also be considered that some patients with CKD need to take glucocorticoids and immunosuppressants. This could explain a high disease severity in patients with CKD. In addition, the patients requiring dialysis treatment constitutes a population with a greater risk of infection ${ }^{55}$.

\section{Diabetes mellitus}

Patients with diabetes mellitus are typically more exposed to infectious pathologies, especially bacterial and viral ones of the lower airways. The mechanisms related to this correlation are not very clear. However, hyperglycemia certainly determines an ineffective antibacterial activity of neutrophils and also complications related to long-term diabetes seem to play an important role. Diabetes mellitus has been indicated as an independent risk factor for the development of severe forms of infection even during $\mathrm{H}_{1 N 1}$ and MERS ${ }^{56}$. A worse outcome was seen in COVID-19 patients with hyperglycemia than in those with euglycemia. However, variability in blood glucose values has been widely documented, and this is due both to viral infection and to the use of corticosteroids. To this regard, the management of hyperglycemia therapy, in critically ill patients, is a crucial factor of evaluation, because in the transition to insulin therapy there may be numerous fluctuations in blood glucose. This is a highly deleterious phenomenon in critically ill patients in general, and, in particular, in patients with COVID-19. In fact, the hypoglycemia enhances the innate immune response of the host with the mobilization of pro-inflammatory monocytes and this has a negative impact on cardiovascular mortality. Conversely hyperglycemia, makes patients more susceptible to infections. Therefore, this glycemic variability is predictive of high mortality in intensive care ${ }^{56}$. Diabetes is an important risk factor for COVID-19 progression and adverse endpoints, and it is important to manage in right way blood glucose, maintaining glycemic variability within 70 to $180 \mathrm{mg} / \mathrm{dl}$. In fact, this strategy is associated with a significant reduction in the composite adverse outcomes and death ${ }^{57}$. The prevalence of diabetes in patients with severe COVID-19 is up to $24 \%{ }^{50}$. However, COVID-19 patients with diabetes were older and had higher inflammation response. In particular, it has been reported that in COVID-19 patients with diabetes, there are increased leukocytes, neutrophils and decreased lymphocytes. Inflammatory indicators are more severe in patients with diabetes, such as CRP, procalcitonin, $\mathrm{IL}-2$ receptor, $\mathrm{IL}-6, \mathrm{IL}-8$ and TNF, it is possible to find even increased level of urea nitrogen, albumin and NT-proBNP. These abnormalities suggest that COVID-19 infection, may be associated with progressive systemic injury in patients with diabetes. However, these findings suggested that COVID-19 patients with diabetes, have more serious viral infection and are more inclined to have a bacterial co-infection. These elements could explain why the survival rate of diabetic COVID-19 patients are lower. In conclusion, patients with severe COVID-19 with diabetes tend to be particularly susceptible to receive mechanical ventilation and intensive care ${ }^{58}$. Some studies showed a complex link between COVID-19 and diabetes. SARSCoV-2 use ACE2 receptor to entry in target cells, and seems that hyperglycemia upregulate the expression of ACE2 receptor, so this could facilitate viral cell entry. The expression of ACE2 on pancreatic $\beta$ cells can lead to a direct effect on $\beta$ cell function, so COVID-19 could induce new onset of diabetes. To this regard, a study showed cases of Diabetic Ketoacidosis (DKA) at the moment of admission in hospital ${ }^{59}$.

\section{Arterial hypertension}

Many studies in literature indicate a close relation between COVID-19 and arterial hypertension. One of these, was conducted on 1099 patients in China, and showed hypertension in $15 \%$ of population with COVID-19, that became $23.5 \%$ in severe patterns of COVID-19. This percentage was more than every other single disorder analyzed as COPD, Diabetes, chronic renal disease, cancer ${ }^{38}$. In a review on May 2020, the prevalence of hypertension ranged from $4.5 \%$ in noncritically ill patients to $>30 \%$ in older patients with COVID-19. Therefore, it not only appears to be associated with COVID-19, but it could be also linked to the rate of severity of the disease. More studies will be needed ${ }^{60}$. Different studies showed an increased risk of death in patient with hypertension ${ }^{37,38,61}$. A recent retrospective study indicates that patients with hypertension have shown a higher incidence of dyspnea and impaired blood markers on admission. They also appear to have needed greater intensity of care ${ }^{62}$. Hypertension has a higher prevalence in the elderly who, as previously mentioned, represent the age group most affected. For the treatment of hypertension in COVID-19 patients, someone initially hypothesized the possibility that ACEls (angiotensin-converting enzyme inhibitors) and ARBs (angiotensin II receptor blockers) could facilitate 
the infection. This theory was supported by studies in which this type of drug caused an increase in the expression of ACE2, a receptor involved in the process of entering the virus into cells ${ }^{63,64}$. However, other authors have shown how, during COVID-19 infection, there is an over-expression of RAS (with suppression of ACE2), which would contribute to the progression of lung damage, promoting inflammatory response and cytokine storm ${ }^{65}$, cell contraction and vasoconstriction ${ }^{66}$ and $\mathrm{NADH} / \mathrm{NADPH}$ oxidase system ${ }^{67}$. For this reason, it could be even protective to take ACEls/ARBs during this disease because it could limit lung damage. For this reason, worldwide recommendations during this emergency period, advise against suspension this type of drugs in patients who already used it ${ }^{67}$. Further studies on this will be necessary.

\section{CONCLUSIONS}

In conclusion, by the data available in literature, we reported that age (older age), comorbidity (in particular diabetes mellitus and hypertension), special condition (such as pregnancy) and male gender are crucial factor for evolution of COVID-19 disease. In fact, older, male and patients with comorbidity are characterized by worse outcome and higher case fatality rate.

\section{ACKNOWLEDGEMENT}

We thank the doctor Laura Leoncini (Director of Hospital of Marcianise, ASL Caserta, Italy) and doctor Cecilia Politi (Director of Internal Medicine Department, Ospedale F. Veneziale, Isernia, ASREM) for your critical revision of our manuscript.

\section{AUTHORS CONTRIBUTIONS}

TC study conception and design, revision of all clinical trials, manuscript drafting; TC, OP, GS revision of all clinical trials, critical revision. GM, GS, GM, LL, CP critical revision. TC study conception and design, critical revision. All authors read and approved the final version of the manuscript.

\section{References}

1 Perlman S. Another decade, another coronavirus. N Engl J Med 2020;382:760.

2 WHO Director-general's opening remarks at the media briefing on COVID-19, 11 February 2020.

3 Coronavirus Resource Center. John Hopkins University \& Medicine.

4 WHO Director-General's opening remarks at the media briefing on COVID-19, 11 March 2020.

5 Gorbalenya AE, Baker SC, Ralph S, et al, Severe acute respiratory syndrome-related coronavirus: the species and its viruses - a statement of the Coronavirus Study Group. Biorxiv 2020. https://doi.org/10.1101/2020.02.07.937862

6 Istituto Superiore di Sanità. Infografica Sorveglianza Integrata COVID-19 in Italia, aggiornamento 13/05/2020.

7 Giwa AL, Desai A, Duca A. Novel 2019 coronavirus SARSCoV-2 (COVID-19): an updated overview for emergency clinicians. Emerg Med Pract 2020;22:1-28.

8 Liu K, Chen Y, Lin R, et al. Clinical features of COVID-19 in elderly patients: a comparison with young and middleaged patients. J Infect 2020;80:e14-8.

9 Sun ZH. Clinical outcomes of COVID-19 in elderly male patients. J Geriatr Cardiol 2020;17:243-5. https://doi. org/10.11909/j.issn.1671-5411.2020.05.010

10 Wu Z, McGoogan JM. Characteristics of and important lessons from the coronavirus disease 2019 (COVID-19) outbreak in China: summary of a report of 72314 cases from the Chinese Center for Disease Control and Prevention. JAMA 2020;Feb 24. https://doi.org/10.1001/jama.2020

11 Shahid Z, Kalayanamitra R, McClafferty B, et al. COVID-19 and older adults: what we know. J Am Geriatr Soc 2020;68:926-9.

12 Lian J, Jin X, Hao S, et al. Analysis of epidemiological and clinical features in older patients with coronavirus disease 2019 (COVID-19) out of Wuhan. Clin Infect Dis 2020;Mar 25:ciaa242.

13 Wang L, He W, Yu X, et al. Coronavirus disease 2019 in elderly patients: characteristics and prognostic factors based on 4-week follow-up. J Infect 2020;80:639-45.

14 Armitage R, Nellums LB. COVID-19 and the consequences of isolating the elderly. Lancet Public Health 2020;5:e256. https://doi.org/10.1016/S2468-2667(20)30061-X

15 Santini Z, Jose P, Cornwell E, et al. Social disconnectedness, perceived isolation, and symptoms of depression and anxiety among older Americans (NSHAP): a longitudinal mediation analysis. Lancet Public Health 2020;5:e6270.

16 Li T, Zhang Y, Gong C, et al. Prevalence of malnutrition and analysis of related factors in elderly patients with COVID-19 in Wuhan, China. Eur J Clin Nutr 2020;74:871-5. https:// doi.org/10.1038/s41430-020-0642-3

17 Sciacqua A, Pujia R, Arturi F, et al. COVID-19 and elderly: beyond the respiratory drama. Intern Emerg Med 2020. https://doi.org/10.1007/s11739-020-02424-x

18 Ludvigsson JF. Systematic review of COVID-19 in children shows milder cases and a better prognosis than adults. Acta Paediatr 2020;00:1-8.

19 Tung Ho CL, Oligbu P, Ojubolamo O, et al. Clinical characteristics of children with COVID-19. AIMS Public Health 2020;7:258-73. https://doi.org/10.3934/publichealth.2020022

20 Dong $\mathrm{Y}, \mathrm{Mo} \mathrm{X}, \mathrm{Hu} \mathrm{Y}$, et al. Epidemiological characteristics of 2143 pediatric patients with 2019 coronavirus disease in China. Pediatrics 2020. [Epub haed of print]

21 Wang Y, Zhu F, Wang C, et al. Children hospitalized with severe COVID-19 in Wuhan. Pediatr Infect Dis J 2020;39:e914. https://doi.org/10.1097/INF.0000000000002739 
22 Xia W, Shao J, Guo Y, et al. Clinical and CT features in pediatric patients with COVID-19 infection: different points from adults. Pediatr Pulmonol 2020;Mar 5. [Epub haed of print]

23 Balasubramanian S, Rao NM, Goenka A, et al. Coronavirus disease 2019 (COVID-19) in children - what we know so far and what we do not. Indian Pediatr 2020;57:435-42. https://doi.org/10.1007/s13312-020-1819-5

24 Riphagen S, Gomez X, Gonzalez-Martinez C, et al. Hyperinflammatory shock in children during COVID-19 pandemic. Lancet 2020;May 7:S0140-6736(20)31094-1

25 Health Alert Network (HAN): Multisystem Inflammatory Syndrome in Children (MIS-C) associated with Coronavirus Disease 2019 (COVID-19) (https://emergency.cdc.gov/ han/2020/han00432.asp).

26 Verdoni L, Mazza A, Gervasoni A, et al. An outbreak of severe Kawasakilike disease at the Italian epicentre of the SARS-CoV-2 epidemic: an observational cohort study. Lancet 2020;May 13. https://doi.org/10.1016/S01406736(20)31103-X

${ }_{27}$ Lu Q, Shi Y. Coronavirus disease (COVID-19) and neonate: what neonatologist need to know. J Med Virol 2020;92:564-7. https://doi.org/10.1002/jmv.25740

28 Qiao J. What are the risks of COVID-19 infection in pregnant women? Lancet 2020;395:760-2.

29 Phoswa WN, Khaliq OP. Is pregnancy a risk factor of COVID-19?, Eur J Obstet Gynecol 2020. https://doi. org/10.1016/j.ejogrb.2020.06.058

30 Chen D, Yang H, Cao Y, et al. Expert consensus for managing pregnant women and neonates born to mothers with suspected or confirmed novel coronavirus (COVID-19) infection. Int J Gynaecol Obstet 2020;149:130-6. https:// doi.org/10.1002/ijgo.13146

${ }_{31}$ Liu H, Liu F, Li J, et al. Clinical and CT imaging features of the COVID-19 pneumonia: focus on pregnant women and children. J Infect 2020;80:e7-13. https://doi.org/10.1016/j. jinf.2020.03.007

32 Qi H, Luo X, Zheng Y, et al. Safe delivery for pregnancies affected by COVID-19. BJOG 2020;127:927-9.

з3 Chen H, Guo J, Wang C, et al. Clinical characteristics and intrauterine vertical transmission potential of COVID-19 infection in nine pregnant women: a retrospective review of medical records. Lancet 2020;395:809-15.

34 Rasmussen SA, Jamieson DJ. Coronavirus disease 2019 (COVID-19) and pregnancy: responding to a rapidly evolving situation. Obstet Gynecol 2020;135:999-1002. https:// doi.org/10.1097/AOG.0000000000003873

35 Gausman J, Langer A. Sex and gender disparities in the COVID-19 pandemic. J Womens Health (Larchmt) 2020;29:465-6.

36 Ambrosino I, Barbagelata E, Ortona E, et al. Gender differences in patients with COVID-19: a narrative review. Monaldi Arch Chest Dis 2020;May 25. https://doi. org/10.4081/monaldi.2020.1389 [Epub haed of print]

37 Wang D, Hu B, Hu C. Clinical characteristics of 138 hospitalized patients with 2019 novel coronavirus-infected pneumonia in Wuhan, China. JAMA 2020;Feb 7. [Epub ahead of print]
38 Guan WJ, Ni ZY, Hu Y. Clinical characteristics of coronavirus disease 2019 in China. N Engl J Med 2020;382:1708-20.

39 Goyal P, Choi JJ, Pinheiro LC, et al. Clinical characteristics of Covid-19 in New York City. N Engl J Med 2020;382:23724. https://doi.org/10.1056/NEJMc2010419.

40 http://globalhealth5050.org/covid19; accessed April 18, 2020.

41 Xie J, Tong Z, Guan X, et al. Clinical characteristics of patients who died of coronavirus disease 2019 in China. JAMA Netw Open 2020;3:e205619.

42 Guan WJ, Ni ZY, Hu Y, et al. Clinical characteristics of coronavirus disease 2019 in China. N Engl J Med 2020. https://doi.org/10.1056/NEJM oa2002032

43 Li L-Q, Huang T, Wang Y-Q, et al. COVID-19 patients' clinical characteristics, discharge rate, and fatality rate of meta-analysis. J Med Virol 2020;1-7.

44 Moulton VR. Review sex hormones in acquired immunity and autoimmune disease. Frontiers Immunol 2018; Oct 4.

45 Ortona E, PierDominici M, Rider V. Editorial sex hormone a and gender differeces in immune responses - center for gender specific medicine. Istituito Superiore di Sanità, Rome, Italy, Department of Biology, Pittsburg State university, Pittsburg, USA, 9 may 2019.

46 Schurz H, Salie M, Tromp G, et al. The X chromosome and sex-specific effects in infectious disease susceptibility. Human Genomics 2019;13:2.

47 Bhatia K, Zimmerman MA, Sullivan JC. Sex differences in angiotensin-converting enzyme modulation of Ang (1-7) levels in normotensive WKY rats. Am J Hypertens 2013;26:591-8.

48 Gebhard C, Regitz-Zagrosek V, Neuhauser HK, et al. Impact of sex and gender on COVID-19 outcomes in Europe. Biol Sex Differ 2020;11:29. https://doi.org/10.1186/ s13293-020-00304-9

49 Cai H. Sex difference and smoking predisposition in patients with COVID-19 [published correction appears in Lancet Respir Med 2020;8:e26]. Lancet Respir Med 2020;8:e20.

50 Liu N, Zhang F, Wei C, et al. Prevalence and predictors of PTSS during COVID-19 outbreak in China hardest-hit areas: gender differences matter. Psychiatry Res 2020;287:112921. https://doi.org/10.1016/j.psychres.2020.112921

51 Oyelade T, Alqahtani J, Canciani G. Prognosis of COVID-19 in patients with liver and kidney diseases: an early systematic review and meta-analysis. Trop Med Infect Dis 2020;5:e80.

52 Boettler T, Newsome PN, Mondelli MU, et al. Care of patients with liver disease during the COVID-19 pandemic: EASL-ESCMID position paper. JHEP Rep 2020;2:100113. https://doi.org/10.1016/j.jhepr.2020.100113

53 Pirola CJ, Sookoian S. SARS-CoV-2 virus and liver expression of host receptors: putative mechanisms of liver involvement in COVID-19. Liver Int 2020;Apr 30. https:// doi.org/10.1111/liv.14500

54 Juan Li, Shuang-Xi Li, Li-Fang Zhao, et al. Management recommendations for patients with chronic kidney disease during the novel coronavirus disease 2019 (COVID-19) 
epidemic. Chronic Dis Transl Med 2020;May 13. [Epub ahead of print]

55 Gentile S, Strollo F, Ceriello A. COVID-19 Infection in italian people with diabetes: lessons learned for our future (an experience to be used), Diabetes Res Clin Pract 2020. [Epub ahead of print]

56 Zhu L, She ZG, Cheng X, et al. Association of blood glucose control and outcomes in patients with COVID-19 and pre-existing type 2 diabetes. Cell Metab 2020;31:106877.e3. https://doi.org/10.1016/j.cmet.2020.04.021

57 Yan $Y$, Yang $Y$, Wang $F$, et al. Clinical characteristics and outcomes of patients with severe COVID-19 with diabetes. BMJ Open Diabetes Res Care 2020;8:e001343. https://doi.org/10.1136/bmjdrc-2020-001343

58 Bornstein SR, Rubino F, Khunti K, et al. Practical recommendations for the management of diabetes in patients with COVID-19. Lancet Diabetes Endocrinol 2020;8:54650. https://doi.org/10.1016/S2213-8587(20)30152-2

59 Tadic M, Cuspidi C, Mancia G, et al. COVID-19, hypertension and cardiovascular diseases: should we change the therapy? Pharmacol Res 2020;158:104906. https://doi. org/10.1016/j.phrs.2020.104906

60 Zhou F, Yu T, Du R, et al. Clinical course and risk factors for mortality of adult inpatients with COVID-19 in Wuhan, China: a retrospective cohort study. Lancet 2020;395:105462. https://doi.org/10.1016/S0140-6736(20)30566-3
61 Zhang P, Zhu L, Cai J, et al. Association of inpatient use of angiotensin-converting enzyme inhibitors and angiotensin ii receptor blockers with mortality among patients with hypertension hospitalized with COVID-19. Circ Res 2020;126:1671-81.

62 Igase M, Kohara K, Nagai T, et al. Increased expression of angiotensin converting enzyme 2 in conjunction with reduction of neointima by angiotensin II type 1 receptor blockade. Hypertens Res 2008;31:553-9.

63 Ferrario CM, Jessup J, Chappell MC, et al. Effect of angiotensin-converting enzyme inhibition and angiotensin II receptor blockers on cardiac angiotensin-converting enzyme 2. Circulation 2005;111:2605-10. https://doi.org/10.1161/ CIRCULATIONAHA.104.510461

64 Rodriguez-Iturbe B, Pons H, Johnson RJ. Role of the immune system in hypertension. Physiol Rev 2017;97:112764. https://doi.org/10.1152/physrev.00031.2016

65 Dhaun N, Webb DJ. Endothelins in cardiovascular biology and therapeutics. Nat Rev Cardiol 2019;16:491-502.

66 Zhang Y, Murugesan P, Huang K, et al. NADPH oxidases and oxidase crosstalk in cardiovascular diseases: novel therapeutic targets. Nat Rev Cardiol 2020;17:170-94.

67 Khashkhusha TR, Chan JSK, Harky A. ACE inhibitors and COVID-19: we don't know yet. J Card Surg 2020;35:11723. 10.1152/physrev.00031.201610.1111/jocs.14582 